\title{
Editorial
}

Rev. Estomatol Herediana. 2013 Jul-Set;23(3):115-6

\section{La importancia de la anatomía en ciencias básicas.}

The importance of basic sciences anatomy.

El debate de la enseñanza de la anatomía humana en las ciencias básicas indica los beneficios en la aplicación clínica, un plan de estudios integrado para el aprendizaje y la retención, hacen hincapié en que la educación se ve reforzada cuando se complementa. No obstante, ha habido grandes debates que preocupan exactamente lo que se debe enseñar y cómo.

Diversas modalidades educativas en los planes de estudio de escuelas de odontología y los cambios en las estrategias curriculares desafían a los anatomistas; si el objetivo es introducir la interactividad y la independencia en las experiencias de aprendizaje, aprendizaje asistido por ordenador, en combinación con la instrucción centrada en el profesor puede ser más beneficioso. El factor más importante en este caso es proporcionar el aprendizaje auto-dirigido con el fin de ampliar los horizontes del estudiante en las prácticas con ayuda de computadora.

La educación superior tiene la responsabilidad social de formar profesionales con una adecuada preparación teórico-práctica que respondan de manera creadora a la solución de los problemas que emergen como necesidad social, y posean un alto grado de conocimiento de las ciencias básicas y sentido ético en su actuación, lo cual les permitirá desempeñarse en diferentes especialidades acordes con las exigencias académicas contemporáneas (1).

"Hoy las universidades deben asumir el reto de poseer creatividad y flexibilidad curricular, mostrar sus avances en la producción intelectual y aplicar los resultados de su producción científica, tanto en la creación de conocimientos como en la producción, la formación del pregrado resulta un eslabón fundamental en la formación de los recursos humanos en salud” (1), a fin de que sean aptos y capaces de cumplir los retos contemporáneos, por ello se impone la necesidad de reforzar la enseñanza de las ciencias básicas en especial la anatomía en la educación superior y en particular en ciencias de la salud.

Desde la década del 2000 "se comenzó a transformar la forma organizativa de enseñanza "conferencia” en las asignaturas básicas biomédicas, al utilizar videos VHS, a partir del curso 2007-2008, videos digitales como medios en las llamadas “conferencias orientadoras (2)”. Sin embargo, existe un educador en el aula, llamado facilitador y no se ha estudiado suficientemente el modelo comunicacional aplicado. A partir del estudio de los medios usados en las conferencias de ciencias básicas. 
"Se concluye que este modelo tiene importancia teórico-práctica para diseñar estrategias de trabajo en colectivo en la forma organizativa de la enseñanza "videoclase" supliendo aquellos aspectos con dificultades de comunicación a partir de reflexiones grupales, guiados por el facilitador, así como actividades de relación interalumnos en la clase” (3), mientras se mejoran las dificultades en los medios, y después que se perfeccionen, para aumentar la comunicación educador-estudiante-profesor de video clases en dicha forma organizativa.

Una parte importante del tiempo que invierten los estudiantes de odontología en su formación, especialmente en el área clínica, corresponde a actividades prácticas alrededor de pacientes, en las cuales se enfrentan a casos clínicos reales. A partir del contacto directo con pacientes, de sus conocimientos previos, de lecturas críticas y reflexivas, de la guía y estímulo por parte de docentes instructores o pares con un nivel más avanzado de formación, podrían estructurar su comprensión médica.

El proceso de formación profesional del odontólogo gira en torno a tres grandes ejes: las ciencias básicas, la salud pública y las rotaciones por las especialidades clínicas. Las ciencias básicas aportan los conocimientos, habilidades y actitudes necesarias para un adecuado aprendizaje del ejercicio clínico.

\section{Mg. Fredy Gutierrez Ventura ${ }^{1, a}$ Editor-Jefe}

\section{REFERENCIAS BIBLIOGRÁFICAS}

1. López R, Pérez N, López G. Algunas consideraciones sobre las formas organizativas de enseñanza en la disciplina Morfofisiología Humana EDUMECENTRO 2013;5(3):1-9.

2. Damiani J. Modelo comunicacional para las videoclases en las ciencias básicas biomédicas. Educ Med Super 2011;25(1): 49-57.

3. Puerto G, Ortega B, Capmany J; Cardona K, Suárez C. Hacia una plataforma de comunicaciones completamente óptica. Rev.fac.ing.univ. Antioquia 2008; July/Sept 45: 148-56.

Facultad de Estomatología. Universidad Peruana Cayetano Heredia. Lima, Perú.

a Magister en Estomatología, Especialista en Ortodoncia y Cirugía Oral y Maxilofacial. 